\title{
Large surgically resected leiomyosarcoma of the liver: a case report
}

\author{
Takashi Maeda ${ }^{1 *}$ D, Kyohei Yugawa', Nao Kinjo', Daisuke Imai', Kensaku Sanefuji', Koto Kawata', Shinichiro Ikeda', \\ Keitaro Edahiro ${ }^{1}$, Kazuki Takeishi ${ }^{2}$, Tomohiro Iguchi ${ }^{2}$, Noboru Harada ${ }^{2}$, Mizuki Ninomiya ${ }^{2}$, Shohei Yamaguchi ${ }^{1}$, \\ Kozo Konishi', Shinichi Tsutsui ${ }^{1}$ and Hiroyuki Matsuda ${ }^{1}$
}

\begin{abstract}
Background: Primary hepatic leiomyosarcoma $(\mathrm{PHL})$ is an extremely rare type of tumor. We herein report a case of a large surgically resected leiomyosarcoma of the liver.

Case presentation: A 69-year-old man with a feeling of epigastric compression was referred for examination of an abdominal mass. He had no history of liver disease or alcohol abuse. Liver function tests indicated Child-Pugh class A. Tumor markers were negative. Computed tomography (CT) and magnetic resonance imaging (MRI) revealed a relatively well-contrasted $12 \times 11 \times 8 \mathrm{~cm}$ tumor with well-defined boundary replacing the lateral segment of the liver alongside multiple intrahepatic metastases. Several nodules up to $12 \mathrm{~mm}$ were found in both lungs, suggestive of metastasis. SUVmax of the liver mass and lung tumor in positron emission tomography were 10.4 and 1.5, respectively. Hepatocellular carcinoma was primarily suspected. Lateral segmentectomy of the liver was performed to confirm diagnosis and prevent tumor rupture. Macroscopically, the lateral segment of the liver had been replaced by a lobular or multinodular tumor with a maximum diameter of $15 \mathrm{~cm}$. In pathological findings, the tumor consisted of bundle-like proliferation of complicated banding spindle-like cells with clear cytoplasm, accompanied by storiform pattern and compressed blood vessels. Nuclear fission images were observed in 8/10 HPF. Partial necrosis was present, with associated venous invasion and intrahepatic metastasis. Immunohistochemical staining for tumor cells revealed desmin, a-smooth muscle actin (aSMA), and h-caldesmon were all positive, informing a final diagnosis of PHL. The postoperative course was uneventful, and he was discharged on the 12th postoperative day.

Conclusions: PHL is a rare malignant disease with relatively poor prognosis. To confirm a diagnosis of $\mathrm{PHL}$, immunohistochemical analysis as well as histopathological findings is important. The preferred treatment is surgical resection, sometimes in combination with adjuvant chemotherapy and radiotherapy. Further studies are needed to elucidate and better understand this uncommon clinical entity.
\end{abstract}

Keywords: Leiomyosarcoma, Liver tumor, Immunohistochemistry

\section{Background}

Primary hepatic leiomyosarcoma (PHL) is an extremely rare tumor, accounting for $0.2-2 \%$ of all primary hepatic malignancies [1-6]. PHL usually arises from intrahepatic vascular structures, bile ducts, or ligamentum teres [7-9]; however, the underlying pathogenetic mechanisms have

\footnotetext{
* Correspondence: tksmaeda9@gmail.com

${ }^{1}$ Department of Surgery, Hiroshima Red Cross Hospital and Atomic-bomb Survivors Hospital, 1-9-6 Senda-machi, Naka-ku, Hiroshima 730-8619, Japan Full list of author information is available at the end of the article
}

not yet been identified. Clinical manifestations of PHL are nonspecific, and tumors are generally asymptomatic until they significantly increase in size $[7,8]$. Herein, we report a case of a 69-year-old male patient with a large surgically resected PHL.

\section{Case presentation}

A 69-year-old man with a feeling of epigastric compression was referred to our hospital for examination of an abdominal mass. His past medical history included 
hyperlipidemia, pulmonary emphysema, and bronchial asthma. He had no history of liver disease, blood transfusion, tattooing, or alcohol abuse. He had smoked 30 cigarettes per day for 35 years. The patient's laboratory findings on admission were as follows: white blood cell count, $7500 / \mu \mathrm{l}$; hemoglobin, $13.7 \mathrm{~g} / \mathrm{dl}$; platelet count, $21.1 \times 10^{4} / \mu \mathrm{l}$; C-reactive protein, $0.5 \mathrm{mg} / \mathrm{dl}$; total protein, $6.7 \mathrm{~g} / \mathrm{dl}$; serum albumin, $4.0 \mathrm{~g} / \mathrm{dl} ; \mathrm{UN}, 11.1 \mathrm{mg} / \mathrm{dl}$; serum creatinine, $0.7 \mathrm{mg} / \mathrm{dl}$; glucose, $86 \mathrm{~g} / \mathrm{dl}$; and HbA1c, $5.4 \%$. Liver function tests revealed total bilirubin, $0.5 \mathrm{mg} / \mathrm{dl}$; serum aspartate aminotransferase, $21 \mathrm{IU} / \mathrm{l}$; serum alanine aminotransferase, 14 IU/l; serum glutamyltransferase, 83 IU/l; prothrombin time, $80.8 \%$; and indocyanine green retention rate at $15 \mathrm{~min}, 8.6 \%$, and indicated Child-Pugh class A. Antibody tests were negative for both hepatitis $B$ surface antigen and hepatitis $C$ virus. The following tumor markers were all within normal ranges: $\alpha$ fetoprotein was $5.7 \mathrm{ng} / \mathrm{ml}$ (normal range $<10 \mathrm{ng} / \mathrm{ml}$ ), PIVKA-II was $18 \mathrm{mAU} / \mathrm{ml}$ (normal range $<40 \mathrm{mAU} / \mathrm{ml}$ ), carcinoembryonic antigen was $1.5 \mathrm{ng} / \mathrm{ml}$ (normal range $<5.0 \mathrm{ng} / \mathrm{ml}$ ), carbohydrate antigen $19-9$ was $4.1 \mathrm{ng} / \mathrm{ml}$ (normal range $<37.0 \mathrm{ng} / \mathrm{ml}$ ), and sIL-2R was $362 \mathrm{U} / \mathrm{ml}$ (normal range 121-613 U/ml). Abdominal computed tomography (CT) (Fig. 1) and magnetic resonance imaging (MRI) (Fig. 2) revealed a relatively well-contrasted $12 \times 11 \times 8 \mathrm{~cm}$ tumor with a well-defined boundary replacing the lateral segment of the liver alongside multiple intrahepatic metastases. In addition, several nodules up to $12 \mathrm{~mm}$ were found in both lungs, suggesting metastasis. Contrast-enhanced CT revealed a hypodense tumor on plain scans, with heterogeneous and peripheral enhancement on early phase and delayed washout on late phase. MRI revealed a homogenous hypointense tumor on Tl-weighted images and a hyperintense one in T2-weighted images without encapsulation. On GdEOB-DTPA-enhanced MRI, the tumor was heterogeneously enhanced during the early phase and weakly enhanced during the late phase. SUVmax of the liver mass and the lung tumor in positron emission tomography (PET) were 10.4 and 1.5 , respectively (Fig. 3). No abnormal accumulation was observed in the lymph nodes, bones, peritoneum, or any other sites. Upper and lower gastrointestinal endoscopic examination detected no abnormal findings. Hepatocellular carcinoma was primarily suspected; however, intrahepatic cholangiocarcinoma or mixed hepatocellular and cholangiocarcinoma were other possible diagnoses. Lateral segmentectomy of the liver was performed to confirm diagnosis and prevent tumor rupture. Operation time was $104 \mathrm{~min}$, with a bleeding volume of $240 \mathrm{ml}$. Macroscopically, the lateral segment of the liver was replaced by a lobular or multinodular tumor with a maximum diameter of $15 \mathrm{~cm}$ (Fig. 4). In pathological findings, the tumor consisted of bundle-like proliferation of complicated banding spindle-like cells with clear cytoplasm, accompanied by storiform pattern and compressed blood vessels (Fig. 5). Hydropic change and hyalinization were observed. Various degrees of nuclear atypia were observed, and nuclear fission images were demonstrated in 8/10 high-power field. Partial necrosis was present, with associated venous invasion and intrahepatic metastasis. Immunohistochemical staining for tumor cells revealed desmin, $\alpha$-smooth muscle actin ( $\alpha$ SMA), and h-caldesmon to be diffusely positive, while c-kit, S100, CD34, CAM5.2, EMA, CD163, and DOG1 were negative (Fig. 6). Ki-67 labeling index of the main tumor was $70 \%$. These findings suggested differentiation into the smooth muscle, informing a final diagnosis of leiomyosarcoma of the liver. No other neoplastic lesion was detected on image examinations such as gastrointestinal endoscopy, CT, MRI, and PET, so the tumor was considered to be primary, not metastatic liver tumor. Postoperative course was uneventful, and he was discharged on the 12th postoperative day. Four weeks after surgery, systemic chemotherapy with doxorubicin $\left(75 \mathrm{mg} / \mathrm{m}^{2}\right)$ was commenced for metastatic tumors of the liver and lung.
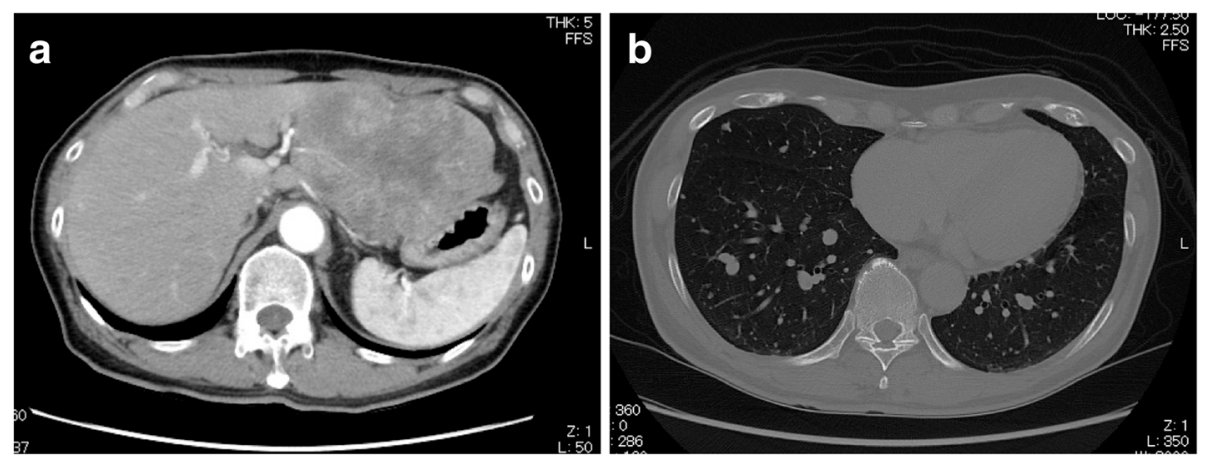

Fig. $1 \mathrm{CT}$ demonstrated a relatively well-contrasted $12 \times 11 \times 8 \mathrm{~cm}$ tumor with well-defined boundary replacing the lateral segment of the liver. Contrast-enhanced CT revealed a heterogeneous and peripheral enhancement on early phase. $\mathbf{b}$ Several nodules up to $12 \mathrm{~mm}$ were observed in both lungs, suggestive of metastasis 


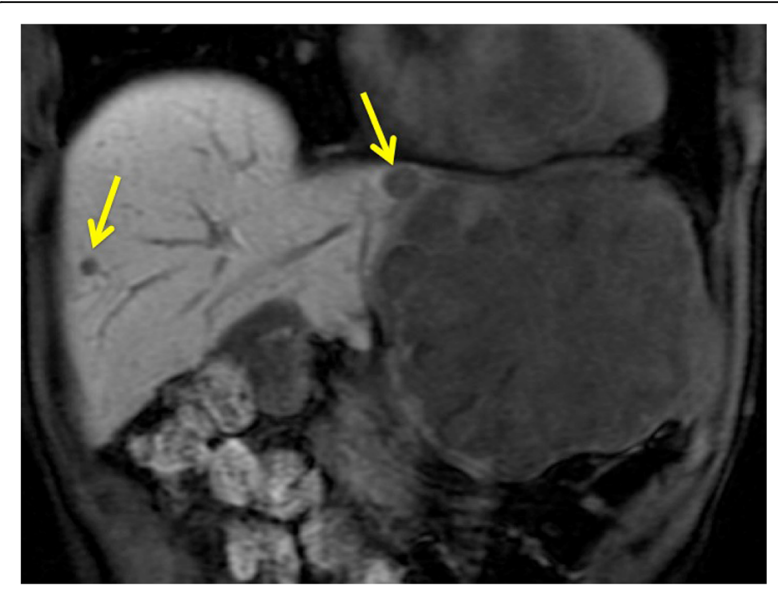

Fig. 2 MRI demonstrated a large tumor and multiple intrahepatic metastases (arrow)

\section{Discussion}

PHL is an extremely rare tumor, accounting for $0.2-2 \%$ of all primary hepatic malignancies [1-6]. PHL usually arises from intrahepatic vascular structures, bile ducts, or ligamentum teres [7-9]. The underlying pathogenetic mechanisms of the disease have not yet been identified; however, it is reportedly associated with AIDS, EpsteinBarr virus, Hodgkin's lymphoma, immunosuppression after organ transplantation, and HCV-related liver cirrhosis $[4,7,10,11]$.

PHL diagnosis is challenging due to its nonspecific symptoms and lack of serological markers. Clinical manifestations and laboratory and imaging examinations have limited value for diagnosis and differential diagnosis. Some patients may present with a wide spectrum of symptoms, including abdominal pain, abdominal mass, weight loss, nausea, vomiting, anorexia, abdominal distension, jaundice, and, rarely, acute intra-abdominal bleeding

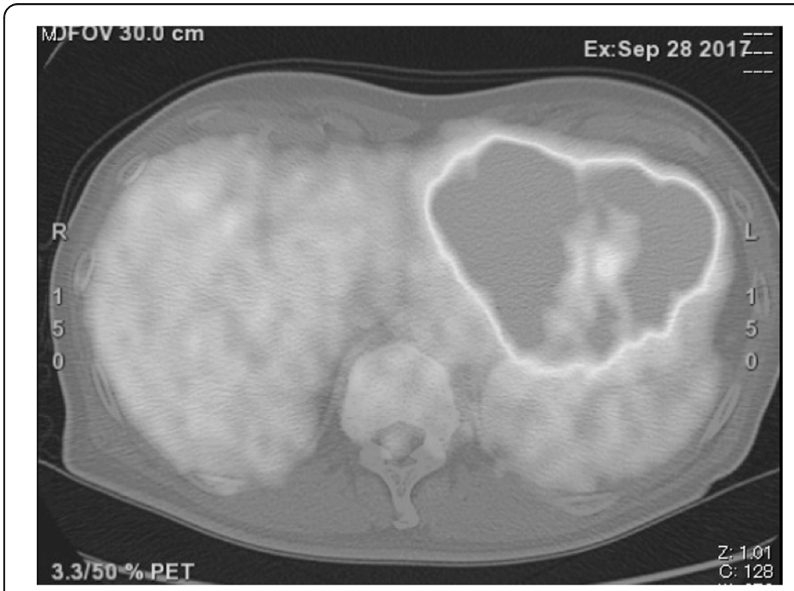

Fig. 3 SUVmax of the liver mass in PET was 10.4. No abnormal accumulation was observed in the lymph nodes, bones, or peritoneum

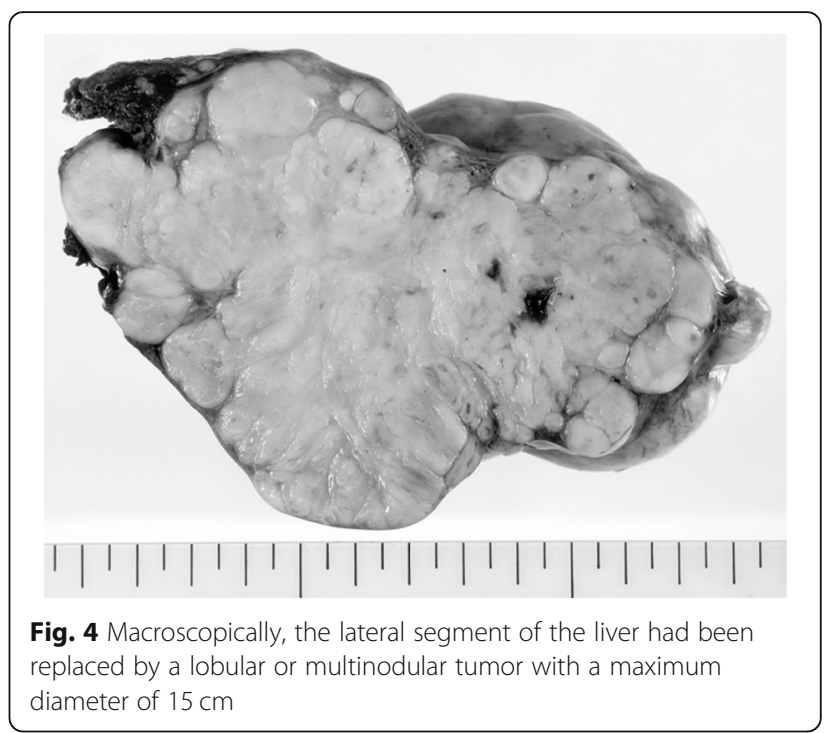

secondary to tumor rupture $[4,7]$. PHL is often asymptomatic until it increases significantly in size, resulting in nonspecific symptoms, or is unexpectedly found during physical examination $[7,8]$. Tumor size at diagnosis varies greatly, with the largest being $30 \mathrm{~cm}$ in diameter. The mean and median diameters for all reported PHLs are $10.3 \mathrm{~cm}$ and $9.1 \mathrm{~cm}$, respectively [4]. In this case, the tumor was detected as a large mass, $15 \mathrm{~cm}$ in diameter, by detailed examination for a feeling of epigastric compression.

Wide ranges of data exist in the literature regarding radiological and histological findings of PHL. Ultrasonography generally shows a hypoechoic mass or a mass with heterogeneous echogenicity [4]. CT findings of PHL generally describe enlarged, heterogeneous, hypodense masses with peripheral enhancement and evidence of central necrosis $[4,7,8,12,13]$, whereas MRI usually displays homogeneous or heterogeneous hypointensity tumors on T1-weighted images and hyperintensity on T2-weighted images [4, 7]. Ferrozzi et al. [12] reported that hemorrhagic necrosis is likely to occur inside the tumor when the tumor grows, and the tumor may show cystic degeneration after necrosis. In this case, the main tumor was large, with heterogeneous and peripheral enhancement on both CT and MRI, suggesting central necrosis of the tumor. Several studies reported that SUVmax of the tumor was correlated with the tumor size, TNM staging, histology subtype, and so on [14-16]. SUVmax of the main tumor in this case was 10.4, much higher than those of metastatic tumor of the liver and lung, which may reflect tumor size. Definitive diagnosis of PHL mainly depends on pathological and immunohistochemical examinations. Histopathological diagnosis of PHL is based on the presence of uniform and diffuse infiltrates of spindle-shaped cells with hyperchromatic nuclei, and presence of mitotic figures $[7,8]$. Positive 

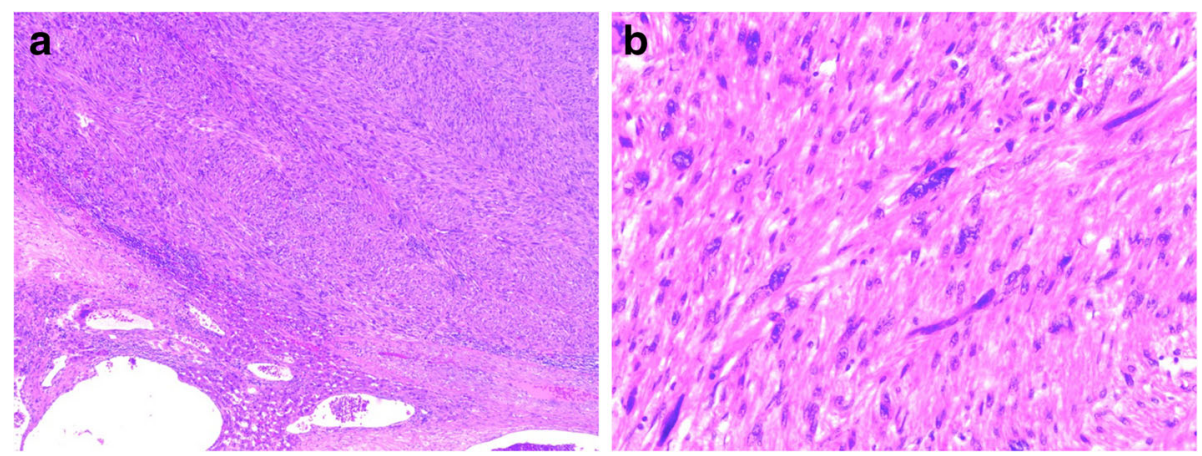

Fig. 5 In pathological findings, a the tumor consisted of bundle-like proliferation of complicated banding spindle-like cells with clear cytoplasm, accompanied by storiform pattern and compressed blood vessels. $\mathbf{b}$ Various degrees of nuclear atypia were observed, and nuclear fission images were demonstrated in 8/10 HPF

immunohistochemistry reactions for $\alpha \mathrm{SMA}$, desmin, and vimentin and negative reaction for cytokeratin, S100 protein as well as $\alpha$-fetoprotein, CD34, CD117, and hepatocytes are used to further confirm diagnosis [4, 7$11,17]$. In this case, tumor cells were positive for desmin, $\alpha \mathrm{SMA}$, and h-caldesmon and negative for c-kit, S100, CD34, CAM5.2, EMA, CD163, and DOG1, supporting the diagnosis of PHL.

Regarding therapeutic options for PHL, hepatic resection is considered the only potentially curative treatment for tumors without distant metastases [4-8]. Matthaei et al. [18] previously reported three cases with $>10$ years survival after hepatectomy. Liver transplantation outcomes for PHL have remained controversial to date $[19,20]$. Furthermore, the efficacies of chemotherapy and radiotherapy for PHL have not been confirmed. Moreover, the role of adjuvant chemotherapy/chemoradiotherapy in PHL is not well defined. Some authors have previously reported the addition of adjuvant chemotherapy consisting of various drug combinations $[2,4,8,13,17,21,22]$. Chemotherapeutic regimens administrated included the following: folinic acid, fluorouracil, irinotecan, and bevacizumab
[17]; mitoxantrone, cisplatin, and fluorouracil [22]; and ifosfamide and mesna [13]. Adjuvant chemotherapy using doxorubicin and ifosfamide seems to slow progression and help to prolong survival after complete resection [9]. Currently, no effective treatments have been reported for unresectable PHL. Fujita et al. [23] reported a patient with metastatic leiomyosarcoma who received only palliative and conservative therapy and survived for 3 months after diagnosis. Radiotherapy has also been used as an adjuvant treatment along with chemotherapy [22, 23]. Finally, transarterial chemoembolization and transarterial infusion of epirubicin and carboplatin have also been reported for treatment $[7,13,21]$.

PHL has aggressive metastatic potential and is usually diagnosed in situations of locally advanced or metastatic disease [5]. Surgical resection is recommended for curative treatment, while diagnosis is challenging and often delayed until reaching a large size, resulting in extremely poor prognosis [7]. Chi et al. [6] reported a median overall survival of 19 months with 1-, 2-, and 5-year survival rates of $61.2 \%, 41.1 \%$, and $14.5 \%$, respectively. They concluded that complete surgical resection with clear

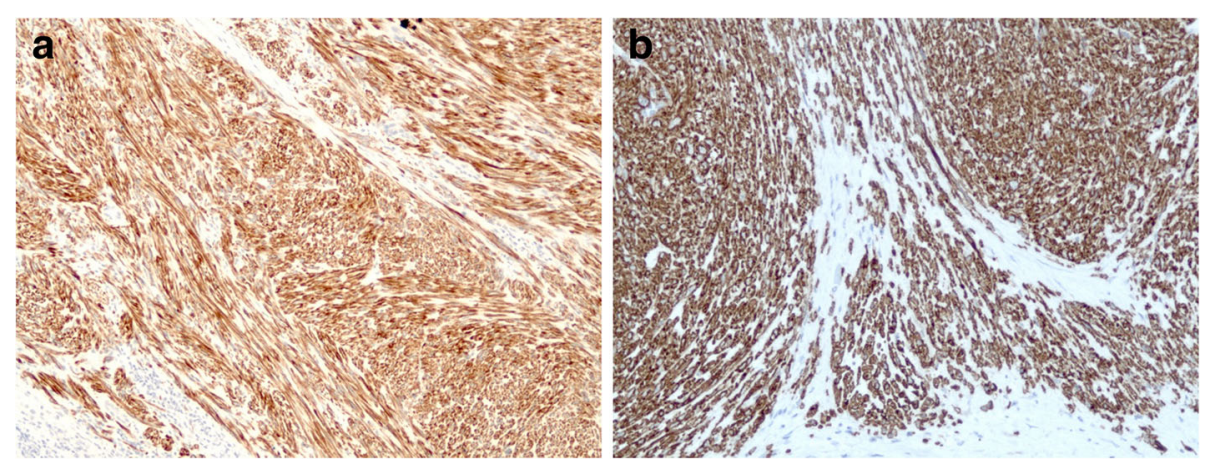

Fig. 6 On immunohistochemical staining, a desmin and aSMA and b h-caldesmon were diffusely positive, while c-kit, S100, CD34, CAM5.2, EMA, CD163, and DOG1 were negative 
margin was necessary to improve survival of patients. In this case, hepatic resection was performed to confirm diagnosis and prevent tumor rupture, although multiple lung nodules, suggesting lung metastases, were found preoperatively.

\section{Conclusions}

In conclusion, PHL is a rare malignant disease with relatively poor prognosis. To confirm PHL diagnosis, immunohistochemical analysis as well as histopathological findings is important. The preferred treatment type is surgical resection, sometimes in combination with adjuvant chemotherapy and/or radiotherapy. Further studies are required to elucidate and better understand this uncommon clinical entity and establish treatment strategies.

\section{Abbreviations}

PHL: Primary hepatic leiomyosarcoma; CT: Computed tomography; MRI: Magnetic resonance imaging; PET: Positron emission tomography; aSMA: a-Smooth muscle actin

\section{Acknowledgements}

The authors would like to thank Enago for the English language review.

\section{Authors' contributions}

TM contributed to the writing of the manuscript. TM, DI, and KS performed the surgery and were responsible for the perioperative management of the patient. ST and HM supervised the study. All authors collected the data and discussed the content of the manuscript. The authors read and approved the final manuscript.

\section{Funding}

No funding was received in support of this work.

\section{Availability of data and materials}

All datasets supporting the conclusions of this article are included in this published article.

\section{Ethics approval and consent to participate}

The present study was conducted in accordance with the ethical standards of the Japan Surgical Society.

\section{Consent for publication}

Written informed consent was obtained from the patient for the publication of this case report and all accompanying images.

\section{Competing interests}

The authors declare that they have no competing interests.

\section{Author details}

${ }^{1}$ Department of Surgery, Hiroshima Red Cross Hospital and Atomic-bomb Survivors Hospital, 1-9-6 Senda-machi, Naka-ku, Hiroshima 730-8619, Japan. ${ }^{2}$ Department of Surgery and Science, Graduate School of Medical Sciences, Kyushu University, 3-1-1 Maidashi, Higashi-ku, Fukuoka 812-8582, Japan.

Received: 15 June 2020 Accepted: 2 July 2020

Published online: 09 July 2020

\section{References}

1. Muranushi R, Hoshino K, Hagiwara K, Yamanaka T, Ishii N, Tsukagoshi M, Igarashi T, Tanaka H, Watanabe A, Kubo N, Araki K, Harimoto N, Ikota H, Shibuya K, Miyazaki M, Shirabe K. Hepatic pleomorphic leiomyosarcoma after surgery for gastric gastrointestinal stromal tumor: a case report. Surg Case Rep. 2019 Dec:5:62.

2. Shamseddine A, Faraj W, Mukherji D, El Majzoub N, Khalife M, Soubra A. Unusually young age distribution of primary hepatic leiomyosarcoma: case series and review of the adult literature. World J Surg Oncol. 2010;8:56.
3. Mitra S, Rathi S, Debi U, Dhiman RK, Das A. Primary hepatic leiomyosarcoma: histopathologist's perspective of a rare case. J Clin Exp Hepatol. 2018;8(3): 321-6.

4. Feretis T, Kostakis ID, Damaskos C, Garmpis N, Mantas D, Nonni A, Kouraklis G, Dimitroulis D. Primary hepatic leiomyosarcoma: a case report and review of the literature. Acta Med (Hradec Kralove). 2018;61(4):153-7.

5. Esposito F, Lim C, Baranes L, Salloum C, Feray C, Calderaro J, Azoulay D. Primary leiomyosarcoma of the liver: two new cases and a systematic review. Ann Hepatobiliary Pancreat Surg. 2020 Feb;24(1):63-7.

6. Chi M, Dudek AZ, Wind KP. Primary hepatic leiomyosarcoma in adults: analysis of prognostic factors. Onkologie. 2012;35:210-4.

7. Zhu KL, Cai XJ. Primary hepatic leiomyosarcoma successfully treated by transcatheter arterial chemoembolization: a case report. World J Clin Cases. 2019;7(4):525-31.

8. Giakoustidis D, Giakoustidis A, Goulopoulos T, Arabatzi N, Kainantidis A, Zaraboukas T. Primary gigantic leiomyosarcoma of the liver treated with portal vein embolization and liver resection. Ann Hepatobiliary Pancreat Surg. 2017;21:228-31.

9. Shivathirthan N, Kita J, Iso Y, Hachiya H, Kyunghwa P, Sawada T, et al. Primary hepatic leiomyosarcoma: case report and literature review. World J Gastrointest Oncol. 2011;3:148-52.

10. Chelimilla H, Badipatla K, Ihimoyan A, Niazi M. A rare occurrence of primary hepatic leiomyosarcoma associated with Epstein Barr virus infection in an AIDS patient. Case Rep Gastrointest Med. 2013;2013:691862.

11. Tsuji M, Takenaka R, Kashihara T, Hadama T, Terada N, Mori H. Primary hepatic leiomyosarcoma in a patient with hepatitis $C$ virus-related liver cirrhosis. Pathol Int. 2000;50(1):41-7.

12. Ferrozzi F, Bova D, Zangrandi A, Garlaschi G. Primary liver leiomyosarcoma: CT appearance. Abdom Imaging. 1996;21:157-60.

13. LV WF, Han JK, Cheng DL, Tang WJ, Lu D. Imaging features of primary hepatic leiomyosarcoma: a case report and review of literature. Oncol Lett. 2015;9:2256-60.

14. Yoh T, Seo S, Morino K, Fuji H, Ikeno Y, Ishii T, Taura K, Nakamoto Y, Higashi T, Kaido T, Uemoto S. Reappraisal of prognostic impact of tumor SUVmax by (18)F-FDG-PET/CT in intrahepatic cholangiocarcinoma. World J Surg. 2019 May;43(5):1323-31.

15. Kitajima K, Yamano T, Fukushima K, Miyoshi Y, Hirota S, Kawanaka Y, Miya M Doi H, Yamakado K, Hirota S. Correlation of the SUVmax of FDG-PET and ADC values of diffusion-weighted MR imaging with pathologic prognostic factors in breast carcinoma. Eur J Radiol. 2016 May;85(5):943-9.

16. Nakamura K, Kodama J, Okumura Y, Hongo A, Kanazawa S, Hiramatsu Y. The SUVmax of 18F-FDG PET correlates with histological grade in endometrial cancer. Int J Gynecol Cancer. 2010 Jan;20(1):110-5.

17. Takehara K, Aoki H, Takehara Y, Yamasaki R, Tanakaya K, Takeuchi H. Primary hepatic leiomyosarcoma with liver metastasis of rectal cancer. World J Gastroenterol. 2012;18:5479-84.

18. Matthaei H, Krieg A, Schmelzle M, Boelke E, Poremba C, Rogiers X, Knoefel WT, Peiper M. Long-term survival after surgery for primary hepatic sarcoma in adults. Arch Surg. 2009;144:339-44.

19. Liang $X$, Xiao-Min S, Jiang-Ping $X$, Jie-Yu Y, Xiao-Jun Z, Zhi-Ren F, Guo-Shan D, Rui-Dong L. Liver transplantation for primary hepatic leiomyosarcoma: a case report and review of the literatures. Med Oncol. 2010;27:1269-72.

20. Saint-Paul MC, Gugenheim J, Hofman P, Arpurt JP, Fabiani P, Michiels JF, Fujita N, Goubeaux B, Loubière R, Delmont J. Leiomyosarcoma of the liver: a case treated by transplantation. Gastroenterol Clin Biol. 1993;17:218-22.

21. Almogy G, Lieberman S, Gips M, et al. Clinical outcomes of surgical resections for primary liver sarcoma in adults: results from a single centre. Eur J Surg Oncol. 2004;30:421-7.

22. Lin YH, Lin CC, Concejero AM, Yong CC, Kuo FY, Wang CC. Surgical experience of adult primary hepatic sarcomas. World J Surg Oncol. 2015;13:87.

23. Fujita H, Kiriyama M, Kawamura T, li T, Takegawa S, Dohba S, Kojima Y, Yoshimura M, Kobayashi A, Ozaki S, Watanabe K. Primary hepatic leiomyosarcoma in a woman after renal transplantation: report of a case. Surg Today. 2002;32:446-9.

\section{Publisher's Note}

Springer Nature remains neutral with regard to jurisdictional claims in published maps and institutional affiliations. 